\title{
Identification of biomarkers and pathways of mitochondria in sepsis patients
}

Gongsheng Yuan*

Department of Physiology and Pathophysiology, School of Basic Medical Sciences,

Fudan University, Shanghai, China

*Corresponding author: Dr. Gongsheng Yuan, Department of Physiology and Pathophysiology, School of Basic Medical Sciences, Fudan University, Shanghai, China gsyuan14@fudan.edu.cn 


\section{Abstract}

Sepsis is a life-threatening condition associate with significant morbidity and mortality, but limited treatment. Mitochondria are recently recognized to be related to the pathophysiology of sepsis, and mitochondria could serve as a potential drug target. In our study, we aim to identify biological functions and pathways of mitochondria during the processes of sepsis by using a bioinformatics method to elucidate their potential pathogenesis. The gene expression profiles of the GSE167914 dataset were originally created by using the Nanostring nCounter Elements ${ }^{\mathrm{TM}}$ TagSet preselected for mitochondrial biogenesis and function panel. The biological pathways were analyzed by the Kyoto Encyclopedia of Genes and Genomes pathway (KEGG), Gene Ontology (GO), and Reactome enrichment. KEGG and GO results showed the Neurodegeneration pathways such as Huntington and Parkinson pathways were mostly affected in the development of sepsis. Moreover, we identified several mitochondrial genes including TOMM40, TOMM20, TIMM22, TIMM10, TIMM17A, TIMM9, TIMM44 were involved in the regulation of protein translocation into mitochondria. Further, we predicted several regulators that had the ability to affect the mitochondria during sepsis by L1000fwd analysis. Thus, this study provides further insights into the mechanism of mitochondrial function during sepsis.

\section{Introduction}

Sepsis is caused by the dysregulated host response to infections from tissue damage or organ dysfunction ${ }^{1}$. Sepsis is a serious disease with a mortality of $15-20 \%$, which is characterized not only by the upregulation of inflammation but also by the strong immune suppression ${ }^{2}$. Understanding the pathophysiology of sepsis may aid in the development of novel therapies ${ }^{3}$. The effects of impaired cellular functions such as mitochondrial dysfunction and cell death mechanism in sepsis-associated organ dysfunction are beginning to be unrevealed ${ }^{4}$.

The significance of mitochondrial dysfunction in sepsis is previously recognized ${ }^{5}$. As the mitochondrion is a critical organelle for multiple cellular processes such as adenosine triphosphate (ATP) production, intracellular calcium homeostasis, and the production of ROS and some hormones ${ }^{6,7}$. Moreover, mitochondria are also associated with 
triggering the intrinsic pathway of apoptosis, which is caused by the outer membrane permeabilization ${ }^{8}$. The mitochondrial functions are changed during sepsis, which includes reduced oxidative phosphorylation, increased ROS production, and altered mitochondrial biogenesis ${ }^{9}$. One hypothesis to explain the dysregulated mitochondrial function is that the decreased oxidative phosphorylation might contribute to the reduced production of potentially harmful $\mathrm{ROS}^{10}$. Mitophagy and mitochondrial biogenesis might be involved in sepsis as a mechanism to reduce the harmful effects of mitochondrial dysfunction ${ }^{5}$. Therefore, during the progression of sepsis, there are various mitochondrial alterations at different times. However, what kind of alteration affects the mitochondrial functions is unclear. Thus, studying the regulation of mitochondria and the related pathways may be a convincing strategy to study the mechanism of sepsis. In this study, we investigated the effect of mitochondrial alterations in sepsis patients. We identified several DEGs, candidate inhibitors, and the relevant biological process in sepsis patients by utilizing comprehensive bioinformatics analyses. The functional enrichment analysis and protein-protein interaction analysis were used for discovering significant gene nodes. These key genes and signaling pathways may be essential to therapeutic interventions of sepsis.

\section{Methods}

Data resources

The GSE167914 dataset was obtained from the GEO database (http://www.ncbi.nlm.nih.gov/geo/). The data was produced by Nanostring nCounter Elements $^{\mathrm{TM}}$ TagSet preselected for mitochondrial biogenesis and function panel, Faculty of Medicine, Universitas Tarumanagara, Jakarta Barat, DKI Jakarta, Indonesia. RNA-Seq analysis was performed using peripheral blood of infection and sepsis patients as well as healthy controls.

Data acquisition and preprocessing

The GSE167914 dataset that contains gene expression related to mitochondrial function from the peripheral blood of infection and sepsis patients as well as healthy 
controls was conducted by $\mathrm{R}$ script $^{11,12}$. We used a classical t test to identify DEGs with $\mathrm{P}<.01$ and fold change $\geq 1.5$ as being statistically significant.

Gene functional analysis

Gene Ontology (GO) is a community-based bioinformatics resource that contains the model Biological Process, Molecular Function, and Cellular Component. Kyoto Encyclopedia of Genes and Genomes (KEGG) database is a useful tool that integrates functional information, biological pathways, and sequence similarity ${ }^{13}$. GO and KEGG pathway analyses were performed by utilizing the Database for Annotation, Visualization, and Integrated Discovery (DAVID) (http://david.ncifcrf.gov/) and Reactome (https://reactome.org/). $\mathrm{P}<0.05$ and gene counts $>10$ were considered statistically significant.

Module analysis

Molecular Complex Detection (MCODE) of Cytoscape software was used to study the connected regions in protein-protein interaction (PPI) networks. The significant modules and clusters were selected from the constructed PPI network using MCODE and String (https://string-db.org/). The pathway enrichment analyses were performed by using Reactome, and $\mathrm{P}<0.05$ was used as the cutoff criterion.

Reactome analysis

The Reactom pathway (https://reactome.org/) was used to obtain the visualization, interpretation, and analysis of potential pathways. $\mathrm{P}<.05$ was considered statistically significant.

\section{Results}

\section{Identification of DEGs of mitochondria from the blood of sepsis patients}

The peripheral blood of sepsis patients and healthy controls were harvested to analyze the DEGs (differentially expressed genes) of mitochondria. Patients fulfilling infection or sepsis criteria were recruited from the emergency department (Faculty of Medicine, Universitas Tarumanagara, Velma Herwanto, Indonesia). A total of 56 genes were 
identified to be differentially expressed in sepsis patients with the threshold of $\mathrm{P}<0.05$. The top 10 up- and down-regulated genes are listed in table 1.

\section{KEGG analysis of DEGs of mitochondria from the blood of sepsis patients}

To identify the biological functions and potential mechanisms of DEGs of mitochondria from sepsis patients and healthy controls, we performed KEGG pathway enrichment analysis and created a visual graph (Supplemental Table S1). KEGG pathway (http://www.genome.jp/kegg/) is a gene collection for exploring the molecular interaction, reaction, and relation networks. Our study showed top ten enriched KEGG pathways including "Neurodegeneration", "Platinum drug resistance", "p53 signaling pathway", "Huntington disease", "Mitophagy", "Apoptosis", "Parkinson disease", "Apoptosismultiple species", "Measles", and "Colorectal cancer" (Figure 1).

\section{GO analysis of DEGs from the blood of sepsis patients}

Gene Ontology (GO) analysis is a commonly used tool for classifying genes, which includes cellular components (CC), molecular functions (MF), and biological processes (BP). Here, we identified the top ten cellular components including "mitochondrial inner membrane", "organelle outer membrane", "outer membrane", "mitochondrial outer membrane", "mitochondrial protein complex", "intrinsic component of organelle membrane", "intrinsic component of mitochondrial membrane", "integral component of organelle membrane", "integral component of mitochondrial membrane", and "intrinsic component of mitochondrial outer membrane" (Figure 1). We then identified the top ten biological processes: "mitochondrial transport", "protein localization to mitochondrion", "establishment of protein localization to mitochondrion", "protein targeting to mitochondrion", "mitochondrial transmembrane transport", "mitochondrial membrane organization", "ATP transport", "adenine nucleotide transport", "purine ribonucleotide transport", and "purine nucleotide transport" (Figure 1). We also identified the top ten molecular functions: "organic anion transmembrane transporter activity", "anion transmembrane transporter activity", "ATP transmembrane transporter activity", "adenine nucleotide transmembrane transporter activity", "purine ribonucleotide transmembrane transporter activity", "purine nucleotide transmembrane transporter 
activity", "nucleotide transmembrane transporter activity", "organophosphate ester transmembrane transporter activity", "nucleobase-containing compound transmembrane transporter activity", and "carbohydrate derivative transmembrane transporter activity" (Figure 2 and Supplemental Table S1).

\section{PPI network and Module analysis}

We constructed PPI networks to analyze the relationships of DGEs at the protein level. The criterion of combined score $>0.7$ was chosen and the PPI network was constructed by using 55 nodes and 106 interactions. Among these nodes, the top ten genes with the highest scores are shown in Table 2. The significant modules of DEGs of mitochondria from the blood of sepsis patients were selected to show the functional annotation (Figure 3).

\section{Reactome Pathway analysis}

To further understand the potential functions of DEGs, we also identified several signaling pathways by using Reactome Pathway Database. The top ten signaling pathways include "Mitochondrial protein import", "Protein localization", "Mitochondrial biogenesis", "Cristae formation", "The citric acid (TCA) cycle and respiratory electron transport", "Formation of ATP by chemiosmotic coupling", "Organelle biogenesis and maintenance", "Intrinsic Pathway for Apoptosis", "Respiratory electron transport, ATP synthesis by chemiosmotic coupling, and heat production by uncoupling proteins", and "Activation of PUMA and translocation to mitochondria" (Supplemental Table S2). We then constructed the visual reaction map according to the signaling pathways (Figure 4).

\section{Potential inhibitors for the treatment of sepsis}

To further know the potential inhibitors for the treatment of sepsis, we introduced the L1000 fireworks display system that can predict bioactive molecules. The system indicated the potential pathways that may be inhibited. We selected the top ten molecules according to the DEGs and the inhibitor map: "BRD-K39757396", "BRDK39829853”, "veliparib”, "BRD-K32862555", "halcinonide”, “DG-041”, “HC-toxin”, “UK356618”, "XMD-1150”, and "GSK-461364" (Figure 5 and Supplemental Table S3). 


\section{Discussion}

Sepsis is a kind of inflammation caused by a serious response of the immune system and finally led to multiorgan failure and death ${ }^{14}$. Sepsis is related to the activation of innate immunity through various pathological processes ${ }^{15}$. For example, the NF-kB signaling pathway as the inflammation center in various diseases ${ }^{16,17}$ is activated by the pathogen-associated molecular patterns (PAMPs) during the beginning of sepsis ${ }^{18}$. Mitochondria provide energy to cells and produce ATP through the basic process of respiration. Numerous cells respond to stresses such as cytokines by triggering mitochondria-dependent signals that initiate cellular protective responses ${ }^{19}$. Thus, based on the mitochondrial study on sepsis patients, our study may provide gene evidence for clinical trials of sepsis.

To understand the effects of mitochondria in sepsis, we analyzed the mitochondrial respiration and gene expression related to mitochondrial functions from the peripheral blood of sepsis patients and healthy controls. Ten proteins were selected according to the PPI network analysis, which may be important during the development of sepsis. TOMM40 is associated with apolipoprotein E (APOE), which further influences agerelated memory performance during Alzheimer's disease ${ }^{20}$. TOMM20 promotes proliferation, resistance to apoptosis, and chemicals ${ }^{21}$. In melanoma cells, enhanced ROS leads to the oxidation and oligomerization of the mitochondrial outer membrane protein Tom $20^{22}$. TIM22 is the complex in the mitochondrial inner membrane ${ }^{23}$. Mitochondrial acylglycerol kinase assembles with TIMM22 to support the import of a subset of multi-spanning membrane proteins ${ }^{24}$. TIMM10 is identified as the blood-based biomarkers for pulmonary tuberculosis by modeling and mining molecular interaction networks ${ }^{25}$. TIM17A is the stress-regulated subunit of the translocase of TIM23, which is downregulated by the eukaryotic initiation factor $2 \alpha(\text { elF2a })^{26}$. TIM9 and TIM44 are responsible for the transport of proteins across the inner membrane ${ }^{27}$.

KEGG and GO analyses indicated that neurodegeneration was the main pathological process during sepsis. The KEGG analysis showed "Neurodegeneration", "Huntington disease", and "Parkinson disease" were the most phenotypes during sepsis, which suggested that the brain or nerve damage was the most affected part during the infection. Similarly, Catherine N Widmann reported that sepsis could cause an increase 
in the permeability of the blood-brain barrier, which may lead to a rapid decline in cognitive function or coma ${ }^{28}$. Thus, sepsis may increase the brain's susceptibility to neurodegenerative disease. Moreover, we also found the cell death-related signaling processes "p53 signaling pathway", "Mitophagy", and "Apoptosis" were involved in sepsis. These signaling pathways were widely enhanced in the damaged cells or cancers $^{29-34}$. Interestingly, these signaling pathways are regulated by the circadian genes such as Clock $^{16,35}$. Circadian clocks play important roles in the physiological and pathophysiological processes ${ }^{36-40}$, such as controlling the immune checkpoint pathways in immune cells ${ }^{41}$. The GO analysis also showed the mitochondrial activity was enhanced during sepsis. Processes like "mitochondrial transport", "protein localization to mitochondrion", "establishment of protein localization to mitochondrion" were associated with the mitochondrial function, which suggested that sepsis could affect the translocation function of mitochondria.

In summary, we identified the potential pathways in sepsis patients by analyzing the mitochondrial gene functions. Neurodegeneration diseases such as Huntington's disease and Parkinson's disease are the mainly triggered diseases during sepsis. Future studies will focus on the administration of potential mitochondrial regulators on clinical trials. This study thus provides further insights into the treatment of sepsis, which may facilitate drug development. 


\section{Reference}

[1] Singer M, Deutschman CS, Seymour CW, Shankar-Hari M, Annane D, Bauer M, Bellomo R, Bernard GR, Chiche JD, Coopersmith CM, Hotchkiss RS, Levy MM, Marshall JC, Martin GS, Opal SM, Rubenfeld GD, van der Poll T, Vincent JL, Angus DC: The Third International Consensus Definitions for Sepsis and Septic Shock (Sepsis-3). JAMA 2016, 315:801-10.

[2] Delano MJ, Ward PA: The immune system's role in sepsis progression, resolution, and long-term outcome. Immunol Rev 2016, 274:330-53.

[3] Gyawali B, Ramakrishna K, Dhamoon AS: Sepsis: The evolution in definition, pathophysiology, and management. SAGE Open Med 2019, 7:2050312119835043.

[4] Exline MC, Crouser ED: Mitochondrial mechanisms of sepsis-induced organ failure. Front Biosci 2008, 13:5030-41.

[5] Singer M: The role of mitochondrial dysfunction in sepsis-induced multi-organ failure. Virulence 2014, 5:66-72.

[6] Zorov DB, Juhaszova M, Sollott SJ: Mitochondrial reactive oxygen species (ROS) and ROS-induced ROS release. Physiol Rev 2014, 94:909-50.

[7] Yuan G, Yang S, Liu M, Yang S: RGS12 is required for the maintenance of mitochondrial function during skeletal development. Cell Discov 2020, 6:59.

[8] Wang C, Youle RJ: The role of mitochondria in apoptosis*. Annu Rev Genet 2009, 43:95-118.

[9] Arulkumaran N, Deutschman CS, Pinsky MR, Zuckerbraun B, Schumacker PT, Gomez H, Gomez A, Murray P, Kellum JA, Workgroup AX: Mitochondrial Function in Sepsis. Shock 2016, 45:271-81.

[10] Nita M, Grzybowski A: The Role of the Reactive Oxygen Species and Oxidative Stress in the Pathomechanism of the Age-Related Ocular Diseases and Other Pathologies of the Anterior and Posterior Eye Segments in Adults. Oxid Med Cell Longev 2016, 2016:3164734.

[11] Hanming G, Wei W, Gongsheng Y: Research Square 2020.

[12] Gu H, Yuan G: Identification of potential biomarkers and inhibitors for SARS-CoV-2 infection. medRxiv 2020:2020.09.15.20195487.

[13] Gu H, Yuan G: Identification of specific biomarkers and pathways in the synovial tissues of patients with osteoarthritis in comparison to rheumatoid arthritis. bioRxiv 2020:2020.10.22.340232.

[14] Bosmann M, Ward PA: The inflammatory response in sepsis. Trends Immunol 2013, 34:129-36.

[15] Cao C, Yu M, Chai Y: Pathological alteration and therapeutic implications of sepsis-induced immune cell apoptosis. Cell Death Dis 2019, 10:782.

[16] Yuan G, Yang S, Yang S, Ng A, Oursler MJ: RGS12 is a critical proinflammatory factor in the pathogenesis of inflammatory arthritis via acting in Cox2-RGS12-NF kappa B pathway activation loop. J Bone Miner Res: WILEY 111 RIVER ST, HOBOKEN 07030-5774, NJ USA, 2019. pp. 147-.

[17] Yuan G, Yang S, Ng A, Fu C, Oursler MJ, Xing L, Yang S: RGS12 Is a Novel Critical NF-kappaB Activator in Inflammatory Arthritis. iScience 2020, 23:101172.

[18] Mogensen TH: Pathogen recognition and inflammatory signaling in innate immune defenses. Clin Microbiol Rev 2009, 22:240-73, Table of Contents.

[19] Hoek JB, Cahill A, Pastorino JG: Alcohol and mitochondria: a dysfunctional relationship. Gastroenterology 2002, 122:2049-63.

[20] Ferencz B, Laukka EJ, Lovden M, Kalpouzos G, Keller L, Graff C, Wahlund LO, Fratiglioni L, Backman L: The influence of APOE and TOMM40 polymorphisms on hippocampal volume and episodic memory in old age. Front Hum Neurosci 2013, 7:198.

[21] Zhou B, Zhang JY, Liu XS, Chen HZ, Ai YL, Cheng K, Sun RY, Zhou D, Han J, Wu Q: Tom20 senses ironactivated ROS signaling to promote melanoma cell pyroptosis. Cell Res 2018, 28:1171-85.

[22] Burke PJ: Mitochondria, Bioenergetics and Apoptosis in Cancer. Trends Cancer 2017, 3:857-70. 
[23] Jensen RE, Dunn CD: Protein import into and across the mitochondrial inner membrane: role of the TIM23 and TIM22 translocons. Biochim Biophys Acta 2002, 1592:25-34.

[24] Vukotic M, Nolte H, Konig T, Saita S, Ananjew M, Kruger M, Tatsuta T, Langer T: Acylglycerol Kinase Mutated in Sengers Syndrome Is a Subunit of the TIM22 Protein Translocase in Mitochondria. Mol Cell 2017, 67:471-83 e7.

[25] Sambarey A, Devaprasad A, Mohan A, Ahmed A, Nayak S, Swaminathan S, D'Souza G, Jesuraj A, Dhar C, Babu S, Vyakarnam A, Chandra N: Unbiased Identification of Blood-based Biomarkers for Pulmonary Tuberculosis by Modeling and Mining Molecular Interaction Networks. EBioMedicine 2017, 15:112-26.

[26] Rainbolt TK, Atanassova N, Genereux JC, Wiseman RL: Stress-regulated translational attenuation adapts mitochondrial protein import through Tim17A degradation. Cell Metab 2013, 18:908-19.

[27] Rassow J, Dekker PJ, van Wilpe S, Meijer M, Soll J: The preprotein translocase of the mitochondrial inner membrane: function and evolution. J Mol Biol 1999, 286:105-20.

[28] Widmann CN, Heneka MT: Long-term cerebral consequences of sepsis. Lancet Neurol 2014, 13:6306.

[29] Moulder DE, Hatoum D, Tay E, Lin Y, McGowan EM: The Roles of p53 in Mitochondrial Dynamics and Cancer Metabolism: The Pendulum between Survival and Death in Breast Cancer? Cancers (Basel) 2018, 10.

[30] Yuan G, Hua B, Cai T, Xu L, Li E, Huang Y, Sun N, Yan Z, Lu C, Qian R: Clock mediates liver senescence by controlling ER stress. Aging 2017, 9:2647-65.

[31] Fu C, Yuan G, Yang ST, Zhang D, Yang S: RGS12 Represses Oral Cancer via the Phosphorylation and SUMOylation of PTEN. J Dent Res 2020:22034520972095.

[32] Fan XF, Wang XR, Yuan GS, Wu DH, Hu LG, Xue F, Gong YS: [Effect of safflower injection on endoplasmic reticulum stress-induced apoptosts in rats with hypoxic pulmonary hypertension]. Zhongguo Ying Yong Sheng Li Xue Za Zhi 2012, 28:561-7.

[33] Yuan G, Hua B, Yang Y, Xu L, Cai T, Sun N, Yan Z, Lu C, Qian R: The Circadian Gene Clock Regulates Bone Formation Via PDIA3. J Bone Miner Res 2017, 32:861-71.

[34] Hanming G: Research Square 2021.

[35] Chen L, Liu S, Tao Y: Regulating tumor suppressor genes: post-translational modifications. Signal Transduct Target Ther 2020, 5:90.

[36] Zhu Z, Hua B, Shang Z, Yuan G, Xu L, Li E, Li X, Sun N, Yan Z, Qian R, Lu C: Altered Clock and Lipid Metabolism-Related Genes in Atherosclerotic Mice Kept with Abnormal Lighting Condition. Biomed Res Int 2016, 2016:5438589.

[37] Cai T, Hua B, Luo D, Xu L, Cheng Q, Yuan G, Yan Z, Sun N, Hua L, Lu C: The circadian protein CLOCK regulates cell metabolism via the mitochondrial carrier SLC25A10. Biochim Biophys Acta Mol Cell Res 2019, 1866:1310-21.

[38] Zhu Z, Xu L, Cai T, Yuan G, Sun N, Lu C, Qian R: Clock represses preadipocytes adipogenesis via GILZ. J Cell Physiol 2018, 233:6028-40.

[39] Xu L, Cheng Q, Hua B, Cai T, Lin J, Yuan G, Yan Z, Li X, Sun N, Lu C, Qian R: Circadian gene Clock regulates mitochondrial morphology and functions by posttranscriptional way. bioRxiv 2018:365452.

[40] Zhu Z, Hua B, Xu L, Yuan G, Li E, Li X, Sun N, Yan Z, Lu C, Qian R: CLOCK promotes 3T3-L1 cell proliferation via Wnt signaling. IUBMB Life 2016, 68:557-68.

[41] Haspel JA, Anafi R, Brown MK, Cermakian N, Depner C, Desplats P, Gelman AE, Haack M, Jelic S, Kim BS, Laposky AD, Lee YC, Mongodin E, Prather AA, Prendergast BJ, Reardon C, Shaw AC, Sengupta $S$, Szentirmai E, Thakkar M, Walker WE, Solt LA: Perfect timing: circadian rhythms, sleep, and immunity - an NIH workshop summary. JCl Insight 2020, 5. 


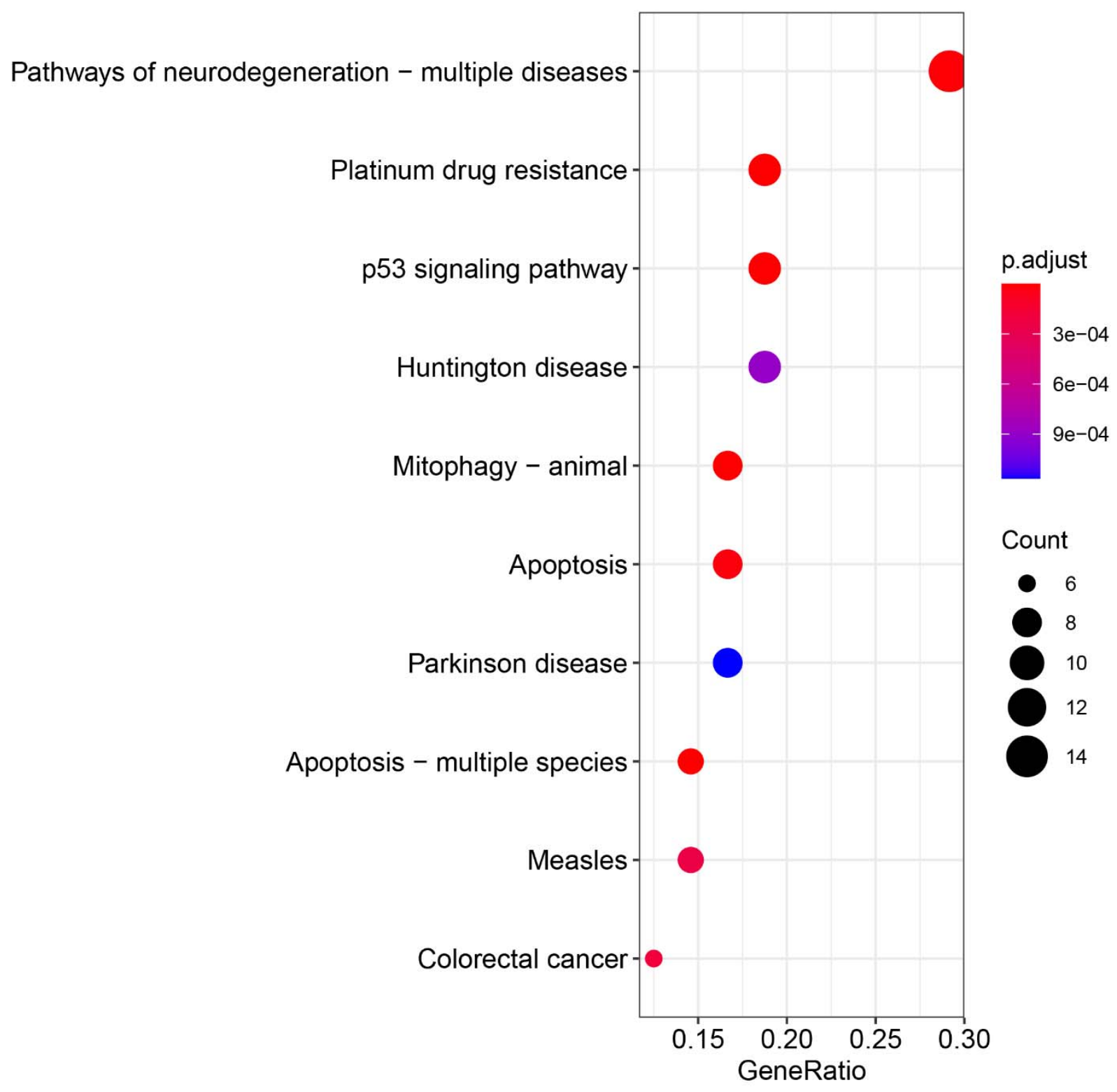

Figure 1. The KEGG pathways enriched by the DEGs. DEGs =differentially expressed genes, KEGG = Kyoto Encyclopedia of Genes and Genomes. 


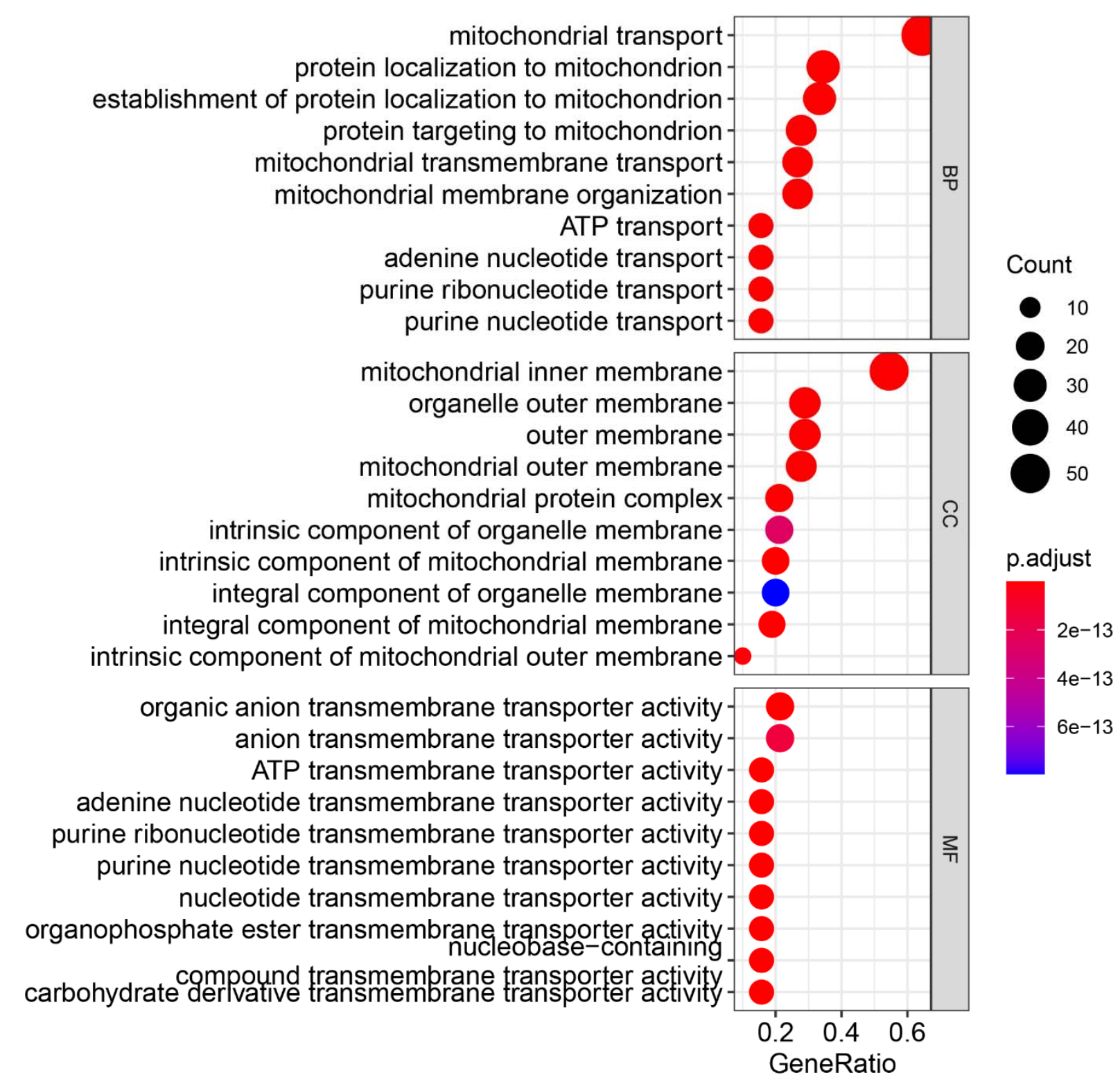

Figure 2. The biological process (BP), cellular component (CC), and molecular function (MF) terms enriched by the DEGs. 


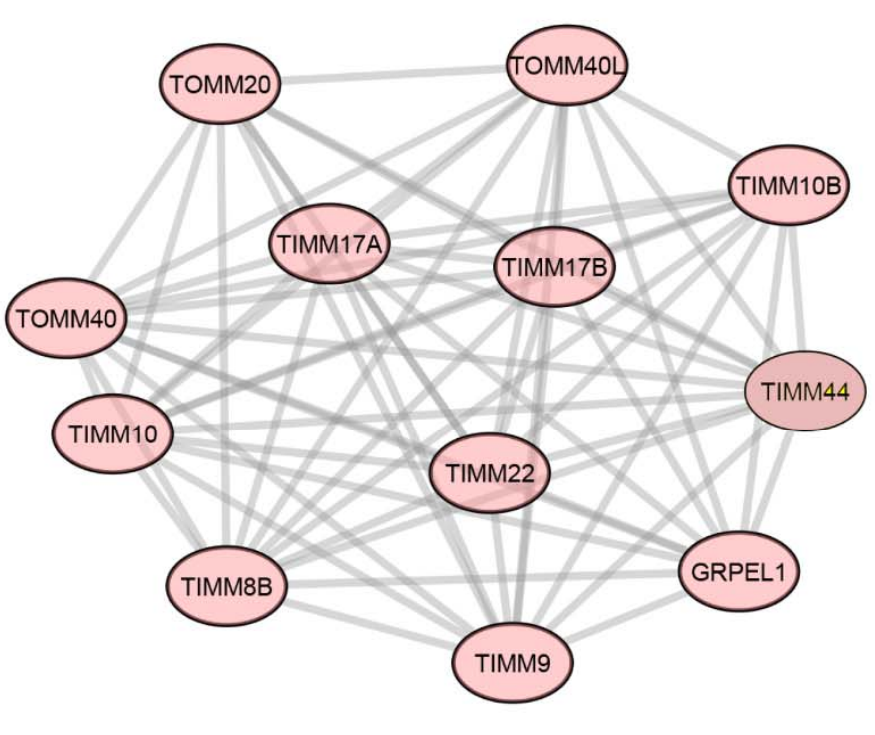

Cluster 1

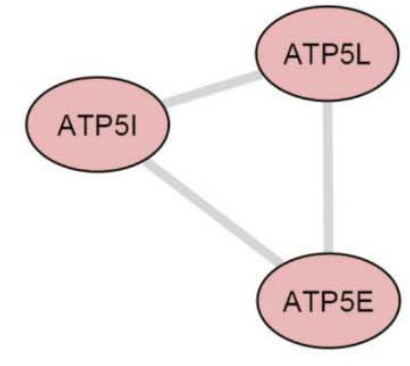

Cluster 2

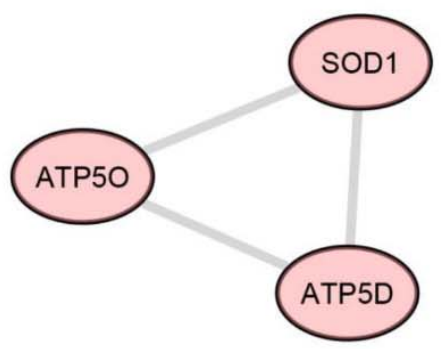

Cluster 3

Figure 3. Top three modules (Cluster1-3) from the blood of sepsis patients 


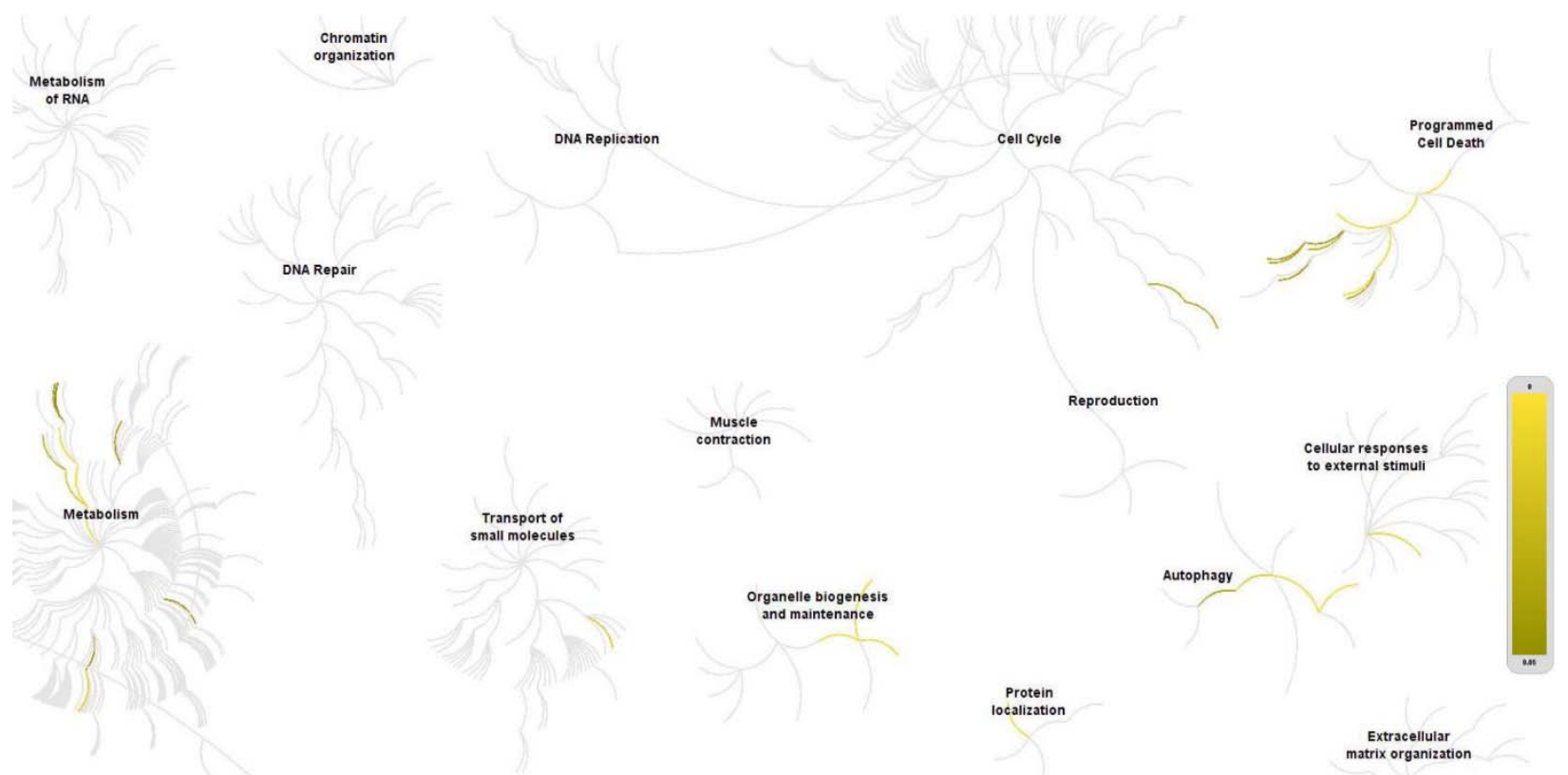

Figure 4. The Reactom pathway visualization map. Input genes are represented by the top significantly changed genes obtained from the GSE167914 dataset $(P<0.01)$. The yellow color represents the most relevant signaling pathways. 


\section{p-value predicted_MOA}

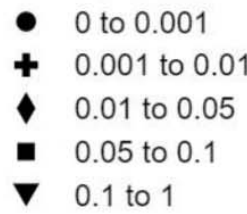

$\boldsymbol{\nabla} 0.1$ to 1
mTOR inhibitor

cyclooxygenase inhibitor

HDAC inhibitor

dopamine receptor antagonist

CDK inhibitor

adrenergic receptor antagonist

topoisomerase inhibitor

NFkB pathway inhibitor

protein synthesis inhibitor

PI3K inhibitor

histamine receptor antagonist glucocorticoid receptor agonist calcium channel blocker

PLK inhibitor

estrogen receptor agonist

other

tubulin polymerization inhibitor

EGFR inhibitor

MEK inhibitor

PARP inhibitor

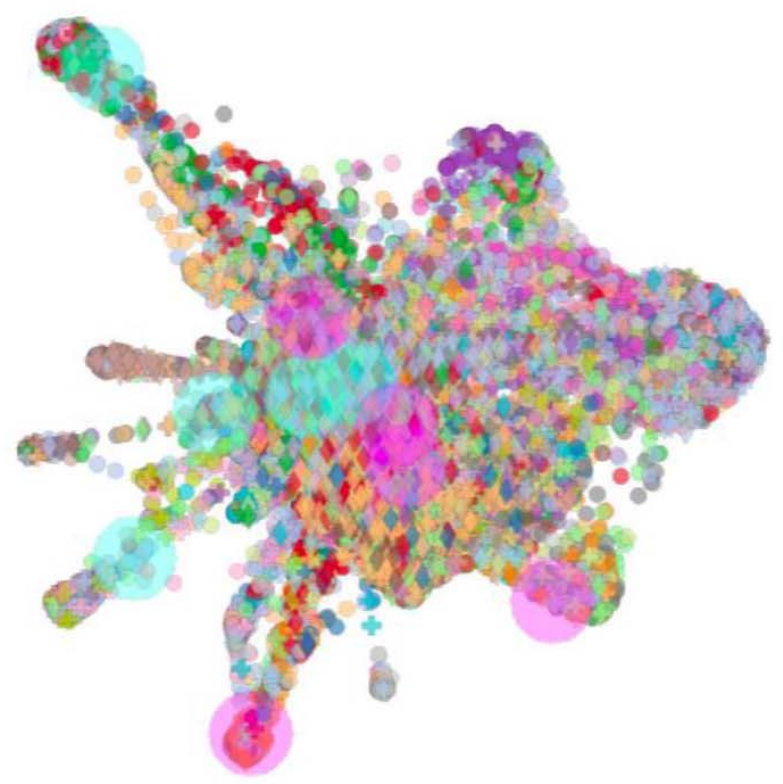

Figure 5. Inhibitors by L1000FDW visualization. Input genes are represented by the significantly changed genes obtained from the GSE167914 dataset. Dots are the Mode of Action (MOA) of the respective drug. 


\section{Table 1}

\begin{tabular}{|c|c|c|c|}
\hline Entrez gene & Gene Symble & Fold-change & Regulation \\
\hline \multicolumn{4}{|c|}{ Top 10 down-regulated DEGs } \\
\hline 706 & TSPO & -1.45828702346694 & Down \\
\hline 26521 & TIMM8B & -1.43210769844688 & Down \\
\hline 51100 & SH3GLB1 & -1.28619452774405 & Down \\
\hline 29957 & SLC25A24 & -1.01302822644703 & Down \\
\hline 55288 & RHOT1 & -1.01271887616278 & Down \\
\hline 788 & SLC25A20 & -0.901108073452252 & Down \\
\hline 84134 & TOMM40L & -0.857069247581503 & Down \\
\hline 10245 & TIMM17B & -0.829410945662233 & Down \\
\hline 3939 & LDHA & -0.800959299777023 & Down \\
\hline 67126 & ATP5E & -0.784378992381854 & Down \\
\hline \multicolumn{4}{|c|}{ Top 10 up-regulated DEGs } \\
\hline 7157 & TP53 & 1.12448582743376 & up \\
\hline 23082 & PPRC1 & 1.03266502881293 & up \\
\hline 596 & BCL2 & 0.994531085428525 & up \\
\hline 66043 & ATP5D & 0.949586740919676 & up \\
\hline 7351 & UCP2 & 0.864981370741031 & up \\
\hline 84172 & POLR1B & 0.728977834365151 & up \\
\hline 3418 & IDH2 & 0.644515388301858 & up \\
\hline 10128 & LRPPRC & 0.642054261768502 & up \\
\hline 23 & ABCF1 & 0.632440787164533 & up \\
\hline 10953 & TOMM34 & 0.623887917139965 & up \\
\hline
\end{tabular}


Table 2. Top ten genes demonstrated by connectivity degree in the PPI network

\begin{tabular}{|c|c|c|}
\hline Gene Symbol & Gene title & Degree \\
\hline TOMM40 & $\begin{array}{l}\text { translocase of outer mitochondrial } \\
\text { membrane } 40\end{array}$ & 16 \\
\hline TOMM20 & $\begin{array}{l}\text { translocase of outer mitochondrial } \\
\text { membrane } 20\end{array}$ & 12 \\
\hline TIMM22 & $\begin{array}{l}\text { translocase of inner mitochondrial } \\
\text { membrane } 22\end{array}$ & 12 \\
\hline TIMM10 & $\begin{array}{l}\text { translocase of inner mitochondrial } \\
\text { membrane } 10\end{array}$ & 12 \\
\hline TIMM17A & $\begin{array}{l}\text { translocase of inner mitochondrial } \\
\text { membrane } 17 \mathrm{~A}\end{array}$ & 11 \\
\hline TIMM17B & $\begin{array}{l}\text { translocase of inner mitochondrial } \\
\text { membrane } 17 \mathrm{~B}\end{array}$ & 11 \\
\hline CYCS & cytochrome c, somatic & 10 \\
\hline TIMM9 & $\begin{array}{l}\text { translocase of inner mitochondrial } \\
\text { membrane } 9\end{array}$ & 9 \\
\hline TIMM44 & $\begin{array}{l}\text { translocase of inner mitochondrial } \\
\text { membrane } 44\end{array}$ & 9 \\
\hline TAZ & tafazzin & 7 \\
\hline
\end{tabular}

